\title{
Multi-Item Distribution Policies with Supply Hub and Lateral Transshipment
}

\author{
Zhong Jin-Hong, Jiang Rui-Xuan, and Zheng Gui \\ School of Management, Hefei University of Technology, Hefei 230009, China \\ Correspondence should be addressed to Zhong Jin-Hong; jinhong.zhong@hfut.edu.cn
}

Received 17 January 2015; Accepted 23 April 2015

Academic Editor: Thomas Hanne

Copyright (c) 2015 Zhong Jin-Hong et al. This is an open access article distributed under the Creative Commons Attribution License, which permits unrestricted use, distribution, and reproduction in any medium, provided the original work is properly cited.

\begin{abstract}
Supply Hub is defined as the horizontal coordination among the suppliers while lateral transshipment is a horizontal coordination policy among the retailers. By considering the Supply Hub and lateral transshipment simultaneously, ones can reduce the total cost of the supply chain system and improve the response to customer requirement and the customers' satisfaction. We investigate the distribution policies for the supply chain which consists of multisuppliers, single Supply Hub, and multidistributors. In the system, both Supply Hub and distributors adopt the $(t, S)$ policy, Supply Hub will not be out of stock, and backlogging is forbidden. Customer requirements at distributors are assumed to be independent random variables complying with uniform distribution; transshipment is assumed to be bidirectional, instantaneous, and emergent. We establish the distribution models, respectively, for the cases of transshipment or no transshipment. For the case with transshipment, we design a GA-based solution method involving a two-stage selection technology, that is, firstly, selecting individuals from parent population to generate offspring chromosomes and, secondly, selecting individuals from the interim population comprising all of the parent and offspring genomes, to form the nextgeneration population. We show that lateral transshipment can increase the overall profit of the supply chain by the comparison examinations between the models with and without transshipment.
\end{abstract}

\section{Introduction}

The main objective of supply chain management (SCM) is to optimize the entire system [1]. Inventory management models of supply chain are the focuses of attention embodying the characteristic of integrated and joint management. Vendor managed inventory (VMI) is a representative model where both supply suppliers and their customers manage their inventory in a win to win manner [2]. VMI is beneficial for suppliers to arrange inventory and production reasonably according to the status of distributors or manufacturers. Moreover, it is helpful to reduce the inventory of the supply and distribution parties, to improve customer satisfaction, so it has been widely applied by firms. Supply Hub mode is the upgrading version of VMI, which allows multiple suppliers to share the storage space of Supply Hub and pool the inventory cost of Supply Hub and is the horizontal cooperation among suppliers. Lateral transshipment is the horizontal collaboration among distributors (retailers), which is profitable to reduce the overall inventory of distributors (retailers) and improve customer satisfaction. Considering lateral transshipment policy under Supply Hub model could improve the flexibility of firms' response to the various customer demands further.

Initially, Supply Hub is aimed at supply chain upstream and is defined as a place which is physically close to manufacturer's facility and is used to store the raw materials of all or some of the suppliers and adopts the agreement that the materials will be paid for only when consumed $[3,4]$. Supply Hub first appears in the practice of enterprise management. Kopczak [5] studies the strategies adopted by Apple computers in the setting up of Supplier Hub at three of their production sites. Zuckerman [3] introduces the analogous practice undertaken by Compaq Computer at its Houston production facility. Barnes et al. [4] point out that Supply Hub evolves gradually from Manufacturer Owned Inventory 
(MOI), VMI, and Supplier Owned Inventory (SOI), particularizes the arguments for and against using Supply Hub, the prerequisite of establishing Supply Hub, and the typical cases of Supply Hub, describes current operation models of Supply Hub, discusses the information technology supporting the operation of Supply Hub, and identified general industry problems and possible research issues. Shah and Goh [6] address the coordination problem in a two-stage supply chain consisting of single supplier, single Supply Hub, and single customer under the deterministic demand of single product, where backlogging is allowed and minimal and maximal inventory limitations at Supply Hub are considered; they develop a structured hierarchy method. Gaonkar and Visvanadham [7] investigate an electronic product supply chain composed of single Supply Hub, $N$ first-tier suppliers, and $N$ second-tier suppliers and establish the linear programming model of coordination scheduling with the limited production and inventory capacity. Li et al. [8] establish decentralized inventory/transportation supply chain model and centered inventory/transportation supply chain model considering Supply Hub, respectively, through comparative analysis; they find that the latter could significantly reduce the system total cost and the observation that the closer the Supply Hub is located to the upstream of supply chain, the more cost will be saved. Furthermore, they also find that the latter could lessen the bullwhip effect better compared with the former. Li et al. [9] establish a mixed integer programming model for the supply chain design with two Supply Hubs and put forward the genetic algorithm to solve it. Huang and $\mathrm{Ma}$ [10] address the coordinated replenishment policy between suppliers considering milk run and Supply Hub and compared it with traditional replenishment policy. Liu and Chen [11] study the single product supply chain comprising multiple manufacturers and multiple retailers under the asymmetric information environment, for the case of the nonlinear relationship between demand and price; they establish the models with and without Supply Hub, respectively. Lin [12] studies the supply chain involving multiple suppliers, single VMI Hub, multiple factories, and multiple customers and compares the two modes of supply inventory managed by firm itself and by VMI Hub. Qiu and Huang [13] put forward the concept of Supply Hub in Industrial Park (SHIP) aimed at warehousing and logistics services and establish the production/distribution models with and without SHIP, respectively, give a genetic algorithm to solve the models, and show that the freight consolidation is profitable to the entire industrial park. Qiu et al. [14] present the thought enhancing the efficiency and effectiveness of the physical assets and services sharing through the SHIP driven by the Internet of things (IOT).

The earliest study of lateral transshipment is about the single period maximal inventory order policy [15]. Up to date, there have been many scholars to conduct extensive research in this area [16-18], the existing research mainly aims at the single product, and the research on multiproduct is scarce. Archibald et al. [19] study multi-item two-location inventory system with lateral transshipment and emergency order, where the periodic review order-up-to $S$ policy is adopted. Kranenburg and van Houtum [20] study multi-item, multilocation, single-echelon spare parts system with base stock control which comprises two types of local warehouse (i.e., main and regular warehouses); only main warehouse can be the supplier of lateral transshipment. Wong et al. [21, 22] study multi-item repairable spare parts inventory system with lateral and emergency shipments where continuous review base stock policy and the average waiting time limitation of required parts are taken into account. They develop a greedy heuristic method which can obtain the approximate optimal solution for multilocation inventory system and a solution procedure based on Lagrangian relaxation for two-location inventory system. Inventory problem of substitutable products can be viewed as lateral transshipment between products. Shumsky and Zhang [23] study the multiperiod dynamic allocation problem of inventory capacity in the firm selling multiple product types of same class, where demand of a product could be met by a product from the next-higher class. Avsar and Baykal-Gürsoy [24] study an infinite period tworetailer inventory control system with two kinds of substitutable products using stochastic game theory. Netessine and Rudi [25] study the centered inventory management model of multiple substitutable products and decentralized inventory management model in which each firm manages a product, respectively. Rottkemper et al. [26] study the inventory relocation and distribution for relief items transshipment network comprising a global, a central, and a number of regional depots, build a multiperiod mixed-integer programming model with minimization of unsatisfied demand and minimization of operational costs, and suggest a rolling horizon solution method. Alvarez et al. [27] consider a multiitem spare parts network of a central depot and multiple local warehouses, where each warehouse has its own premium and nonpremium customers with class-specific waiting time restrictions; lateral transshipment and emergency shipments are used as differentiation tool. They develop a heuristic approach similar to Dantzig-Wolfe decomposition to set stock levels and (trans)shipment strategies of multi-item system. Moghaddam and Nof [28] investigate demand and capacity sharing decisions problem in collaborative network of supply enterprises under uncertain multi-item demand, where the best matching protocol is proposed to minimize the total cost of collaboration and the gap between the capacity of the supply enterprises and their allocated demands. They build a fuzzy mixed-integer planning model for the problem.

Although many authors have widely applied GA to solve SCM problems, we cannot find the research which is directly related to this work. So we shall provide an overview for the literature on the inventory management for one-to-many supply chain under VMI mode and multilocation inventory system. For the VMI system, Michaelraj and Shahabudeen [29] research optimal replenishment policies of the distributors working in the scenario that retailers are making the payment to the distributors in number of unequal installments and use GA and the linear programming to solve the a distributor, multiretailer single item multiperiod VMI system to minimize balance payment and maximize sales. Lan et al. [30] establish two inventory control models of deteriorating item for the single item multiperiod supply chain including a manufacturer, a vendor, and many retailers located in 
different regions, respectively, according to the time-based and the quantity-based integrated delivery strategies for suppliers under VMI model and design a GA to resolve the models. Nachiappan and Jawahar [31] present a GA-based heuristic to deal with the operational issues of single vendormultiple buyers, single item supply chain model under VMI mode of operation. Sue-Ann et al. [32] research the operational issues of a single vendor/multiple buyers single item supply chain under VMI mode and propose a hybrid of GA and Artificial Immune System to solve the problem. Diabat [33] addresses the issue of VMI by considering a single vendor/multiple buyers single item supply chain network and develops a hybrid genetic/simulated annealing algorithm to cope with the nonlinear problem. Sadeghi et al. [34] establish a biobjective single item VMI model in a supply chain with one vendor and several retailers, where the constraints are the total budget, required storage space, vendor's total replenishment frequencies, and average inventory; they employ a nondominated sorting genetic algorithm-II (NSGA-II) to minimize the total system inventory cost and the total system reliability. Later, Sadeghi and Niaki [35] consider the other two objectives of the minimization of the total inventory cost and the warehouse space for the same SC and develop a NSGA-II as well to solve it. For the multilocation system, Chan et al. [36] address single item production and distribution problems in multifactory multicustomer supply chain and develop a hybrid GA to determine demands allocation to suitable manufacturers. Maiti et al. [37] study a multi-item two-storage multiple price breaks deterministic inventory model with a discount policy, where the stocks of rented warehouse are transported to the owned warehouse in bulkrelease rule and develop a real-coded GA with advanced GA operators. Pasandideh et al. [38] deal with a two-echelon inventory system for nonrepairable items where the system consists of one warehouse and multiple identical retailers and uses continuous review $(R, Q)$ ordering policy and develop a parameter-tuned genetic algorithm to minimize the total annual inventory investment. Yang et al. [39] suggest GAbased approach to resolve the optimal problem for a multiproduct multiperiod supply chain system of multisupplier, single warehouse and multiretailer with backlogging and transportation capacity. Paul and Rajendran [40] address the problem of determining the inventory control-policy or order-policy parameters and rationing mechanisms for a static divergent one distributor-many retailer multiperiod single item supply chain in a lost sales environment and present a GA-based heuristic methodology.

This study concentrates on multiple products distribution policy considering Supply Hub and lateral transshipment simultaneously. To the best of our knowledge, it still holds open. The sole relative research is the work of Chen et al. [41]. They addressed vendor's distribution policy with transshipment under the VMI environment, for a centralized two-echelon supply chain with one vendor and two retailers selling products with a short life cycle and facing stochastic demand.

The rest of the paper is organized as follows. Section 2 gives the problem description and hypothesis. Section 3 presents multi-item distribution model without

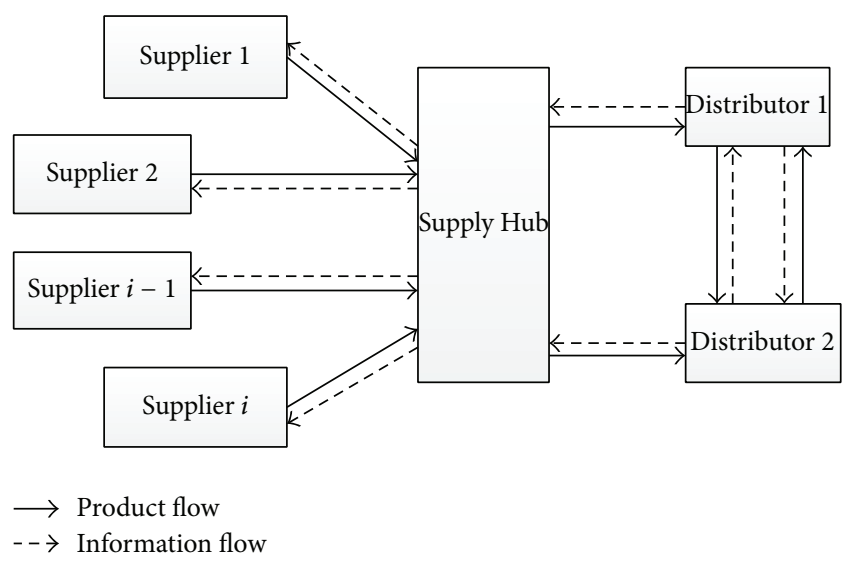

FIGURE 1: Product distribution supply chain considering Supply Hub and lateral transshipment.

transshipment. Section 4 provides multi-item distribution model with transshipment. Section 5 gives a GA-based algorithm to solve the transshipment model. Section 6 is computation example. Section 7 concludes this study.

\section{Problem Description and Hypothesis}

We consider a three-echelon supply chain consisting of multiple suppliers, a Supply Hub, and two distributors (see Figure 1). Each supplier supplies only one product to the Supply Hub, which holds a certain stock to supply the distributors. The demand of supplier is random but complies with uniform distribution in each period. During the replenishment period, lateral transshipment between distributors could be operated to balance their own inventory in order to lessen the overhigh stock or stockout.

For the supply chain as shown in Figure 1, we make the following assumptions.

Assumption 1. The supply chain is composed of multiple suppliers, Supply Hub, and two distributors where each supplier supplies only one product to the Supply Hub.

Assumption 2. The transportation cost comprises fixed charge and unit cost.

Assumption 3. Both Supply Hub and distributor adopt the ( $t$, S) policy.

Assumption 4. The inventory review cycle of Supply Hub is $M$ times of that of the distributor ( $M$ is an integer greater than $1)$.

Assumption 5. The replenishment lead time of Supply Hub is not more than that of distributors; that is to say, Supply Hub will not be out of stock.

Assumption 6. The demand at distributors is independent random variable of uniform distribution at each period.

Assumption 7. Backlogging is not allowed. 
Assumption 8. The demand quantity within lead time can be determined when distributors place replenishment orders; accordingly, two distributors could reduce overhigh stock or stockout.

Assumption 9. Lateral transshipment can be completed instantaneously. This assumption is reasonable in real world. Lateral transshipment commonly takes place between the retailers who are physically very close; it takes very short time (e.g., within one hour) to ship goods from a retailer to another one, while regular replenishment time may be several days or longer. Therefore, transshipment time usually is ignored in the literature on the transshipment, especially for the general or seasonal product; only in some studies of inventory system on service parts, spare parts, repairable items, and expensive low-demand items transshipment time was considered.

Define the following notations to describe the supply chain system:

$i$ : supply (or product) number, $i \in\{1,2, \ldots, I\}$.

$j$ : the replenishment period number of Supply Hub, $j \in\{1,2, \ldots, J\}$.

$\tau$ : the replenishment period number of distributors, $\tau \in\{1,2, \ldots, M J\}$.

$n$ : distributors number, $n \in\{1,2, \ldots, N\}$.

$P_{i}$ : unit procurement cost of product $i$ at Supply Hub.

$F_{i}$ : the fixed cost transporting the product $i$ to Supply Hub.

$A_{i}$ : the unit cost transporting the product $i$ to Supply Hub.

$H_{i}$ : the unit holding cost of product $i$ at Supply Hub.

$T$ : the length of replenishment cycle at Supply Hub.

$L$ : the length of replenishment lead time at Supply Hub.

$Q_{i j}$ : replenishment quantity of product $i$ in replenishment period $j$ at Supply Hub.

$S_{i}$ : the maximal stock level of product $i$ at Supply Hub. $V_{i n}$ : sale price of product $i$ at distributor $n$.

$f_{\text {in }}$ : fixed costs transporting product $i$ to distributor $n$. $a_{i n}$ : unit cost transporting product $i$ to distributor $n$.

$h_{i}$ : unit holding cost of product $i$, which is the same for the same product in different distributor.

LD: the length of replenishment lead time at distributor.

$I_{i \tau n}$ : initial inventory level of product $i$ at distributor $n$ in period $\tau$.

$d_{i \tau n}$ : the demand rate of product $i$ at distributor $n$ in period $\tau$.

$Q_{i \tau n}$ : replenishment quantity of product $i$ at distributor $n$ in period $\tau$.

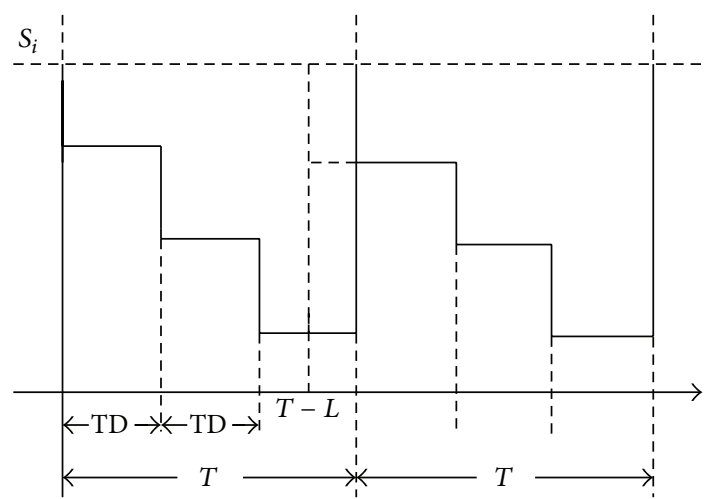

FIGURE 2: The change of inventory level of product $i$ at Supply Hub.

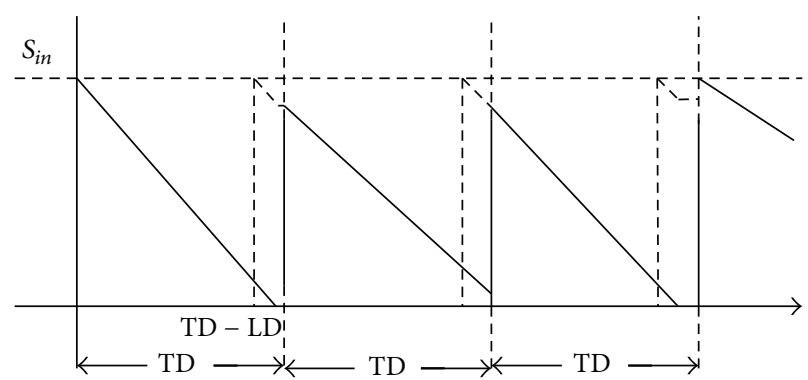

FIGURE 3: The change of inventory level for product $i$ at distributor $n$.

$Q_{i \tau}$ : total replenishment quantity of product $i$ in period $\tau$.

$b_{i}$ : unit shortage cost of product $i$.

$\pi_{i}$ : unit lateral transshipment cost of product $i$.

$S_{i \tau n}$ : the inventory level of product $i$ at distributor $n$ at the outset of period $\tau$.

$\widehat{S}_{i \tau n}$ : inventory level of product $i$ when distributor $n$ place an order in period $\tau$.

$S_{i n}$ : the maximal inventory level of product $i$ at distributor $n$.

\section{The Multi-Item Distribution Model without Lateral Transshipment}

The supplier $i$ supplies the goods of $Q_{i j}$ to Supply Hub every $T$ time; Supply Hub supplies the commodity of $Q_{i \tau n}$ to other distributors every TD time. The changes of inventory level at Supply Hub and distributors are showed in Figures 2 and 3, respectively.

From Figures 2 and 3, we can know that the inventory level of Supply Hub and distributors are replenished to their maximal inventory levels at the beginning of the cycle. The inventory level will decrease with the occurrence of demand. When the time of $(T-L)$ or $(\mathrm{TD}-\mathrm{LD})$ arrives, order will be placed according to the current inventory level and maximal inventory level. 
In the case of no lateral transshipment, the maximal total profit model of the supply chain system is as follows:

$$
\begin{aligned}
& \operatorname{Max} \quad\left\{\mathrm{TS}-\left(\mathrm{TP}+\mathrm{TT}+\mathrm{TI}_{s}+\mathrm{TI}_{d}+\mathrm{TO}\right)\right\} \\
& \text { s.t. } \quad \mathrm{TS}=\sum_{i=1}^{I} \sum_{\tau=1}^{M J} \sum_{n=1}^{N} \min \left(\mathrm{TD} \cdot d_{i \tau n}, S_{i \tau n}\right) \cdot V_{i n} \\
& \mathrm{TP}=\sum_{i=1}^{I} \sum_{j=1}^{J} P_{i} Q_{i j} \\
& \mathrm{TT}=\sum_{i=1}^{I}\left\{\sum_{j=1}^{J}\left(F_{i}+A_{i} \cdot Q_{i j}\right)+\sum_{\tau=1}^{M J} \sum_{n=1}^{N}\left(f_{i n}+a_{i n} \cdot Q_{i \tau n}\right)\right\} \\
& \mathrm{TI}_{s}=\sum_{i=1}^{I} \sum_{j=1}^{J}\left\{\left(S i-\frac{1}{M} \sum_{\tau=M(j-1)+1}^{M j}(M j-\tau+1) \cdot Q_{i \tau}\right) \cdot T \cdot H_{i}\right\} \\
& \mathrm{TI}_{d}= \begin{cases}\sum_{i=1}^{I} \sum_{\tau=1}^{M J} \sum_{n=1}^{N}\left(\frac{S_{i \tau n}^{2} \cdot h_{i}}{2 d_{i \tau n}}\right), & \text { if } d_{i \tau n}<\frac{S_{i \tau n}}{\mathrm{TD}} \\
\sum_{i=1}^{I} \sum_{\tau=1}^{M J} \sum_{n=1}^{N}\left(\begin{array}{ll}
S_{i \tau n} \cdot \mathrm{TD} \cdot h_{i}- \\
\frac{\mathrm{TD}^{2} \cdot d_{i \tau n} \cdot h_{i}}{2}
\end{array}\right), & \text { otherwise }\end{cases} \\
& \mathrm{TO}=\sum_{i=1}^{I} \sum_{\tau=1}^{M J} \sum_{n=1}^{N}\left(\left[\mathrm{TD} \cdot d_{i \tau n}-S_{i \tau n}\right]^{+} \cdot b_{i}\right) \\
& Q_{i j}=\sum_{\tau=M(j-1)+1}^{M j} Q_{i \tau} \\
& Q_{i \tau}=\sum_{n=1}^{N} Q_{i \tau n}
\end{aligned}
$$

where $[x]^{+}$represents that $[x]^{+}=x$ if $x>0 ;[x]^{+}=0$ otherwise. All of the parameters involved in the model are positive integers. The optimization objection (1) is to maximize the total profit of supply chain system. Equations (2)(7) denotes the total sales (TS), the total ordering cost (TP), the total transportation cost (TT), the total holding cost at Supply Hub $\left(\mathrm{TT}_{s}\right)$, the total holding cost at distributors $\left(\mathrm{TT}_{d}\right)$, and the total shortage cost (TO), respectively. Equation (4) involves the transportation cost from suppliers to Supply Hub and one from Supply Hub to distributors. The two cases in (6), respectively, consider two cases where distributor is in stock or out of stock in period $\tau$. Due to the assumption with no shortage at Supply Hub, only stockout cost at distributor is considered in (7).

Note that the calculation of $Q_{i \tau n}$ is the key to solve the model. In view of no backlogging and stock control policy, we can obtain

$$
Q_{i \tau n}=S_{i n}-\widehat{S}_{i(\tau-1) n}
$$

$$
\begin{aligned}
& \widehat{S}_{i \tau n}=\left[S_{i \tau n}-(\mathrm{TD}-\mathrm{LD}) \cdot d_{i \tau n}\right]^{+} \\
& S_{i \tau n}=S_{i n}-\min \left(\widehat{S}_{i(\tau-1) n}, \mathrm{LD} \cdot d_{i(\tau-1) n}\right) .
\end{aligned}
$$

\section{The Multi-Item Distribution Model with Lateral Transshipment}

For the case with lateral transshipment, at time (TD LD) the distributors need to make a decision whether lateral transshipment occurs or not, and how much the transshipment level of each product should be, in order to cut down the unbalanced distribution of the distributors' inventory with respect to their demand. Note that lateral transshipment occurs solely when the inventory level of a distributor is greater than the demand within lead time and status of another distributor is just opposite. Let $X_{i \tau n n^{\prime}}$ be the transshipment amount of product $i$ from distributor $n$ to 

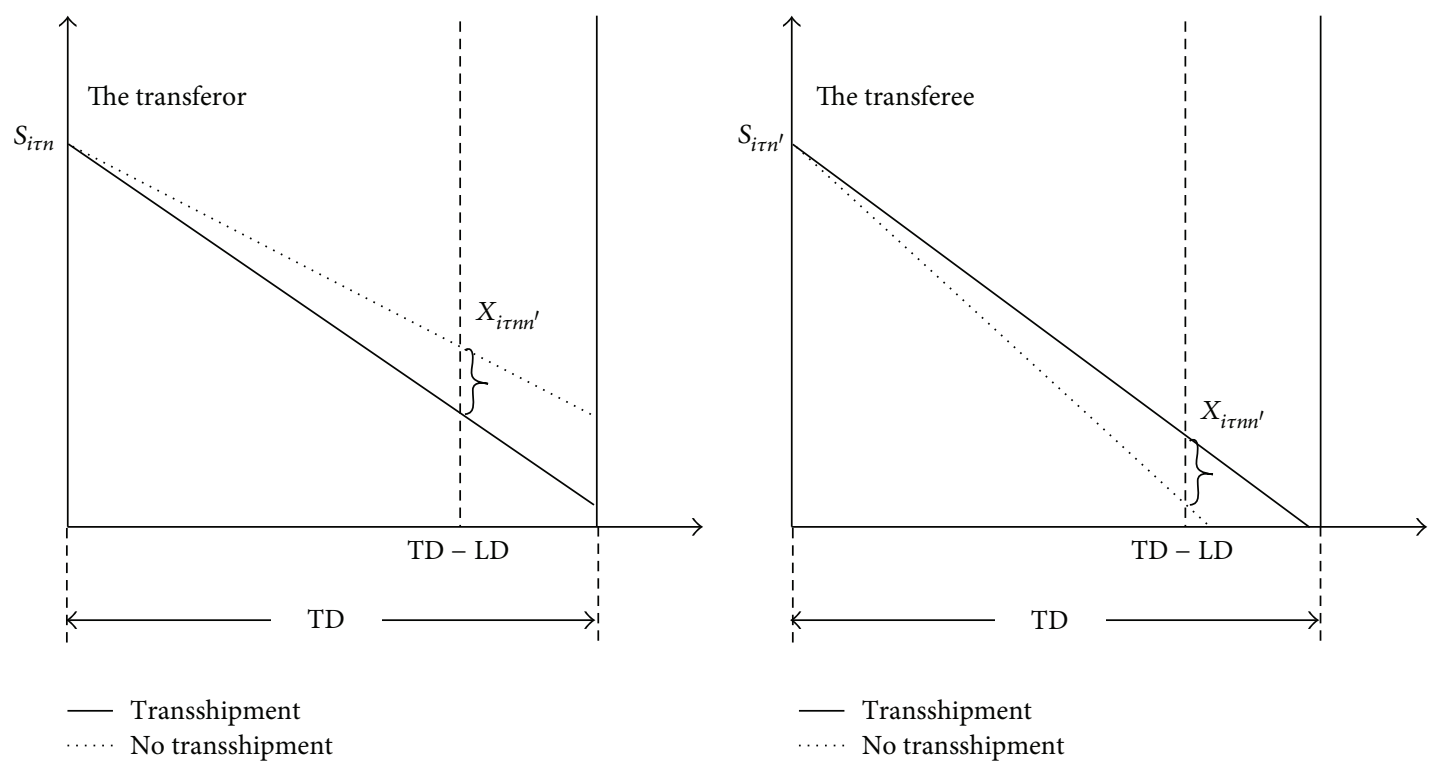

FIGURE 4: The inventory level change of distributor before and after transshipment.

distributor $n^{\prime}$ in period $\tau ; X_{i \tau n^{\prime} n}$ is the transshipment amount of product $i$ from distributor $n^{\prime}$ to distributor $n$ in period $\tau$

$$
\begin{aligned}
& X_{i \tau n n^{\prime}}=\min \left\{\left[\widehat{S}_{i n \tau-1}-\mathrm{LD} \cdot d_{i n \tau-1}\right]^{+},\right. \\
& \left.\left[\mathrm{LD} \cdot d_{i n^{\prime} \tau-1}-\widehat{S}_{i n^{\prime} \tau-1}\right]^{+}\right\} \\
& X_{i \tau n^{\prime} n}=\min \left\{\left[\mathrm{LD} \cdot d_{i n \tau-1}-\widehat{S}_{i n \tau-1}\right]^{+},\left[\widehat{S}_{i n^{\prime} \tau-1}-\mathrm{LD}\right.\right. \\
& \left.\left.\cdot d_{i n^{\prime} \tau-1}\right]^{+}\right\} .
\end{aligned}
$$

Therefore, the total transfer cost (TL) is

$$
\mathrm{TL}=\sum_{\tau=1}^{M} \sum_{i=1}^{I} \sum_{n=1}^{N} \sum_{n^{\prime}=1, n^{\prime} \neq n}^{N} \pi_{i} \cdot\left(X_{i \tau n n^{\prime}}+X_{i \tau n^{\prime} n}\right) .
$$

Note that the transshipment amount from another distributor at most is equal to the stockout level of a distributor within its lead time; that is, it is used to satisfy the demand within lead time. So the transshipment amount should not be counted into transferee's current inventory; the transferee's inventory level at the outset of the next cycle is still calculated by formula (12). But lateral transshipment will affect the beginning inventory of the transferor in the next cycle. If distributor $n$ is the transferor, there is

$$
\begin{aligned}
& S_{i \tau n} \\
& =S_{i n} \\
& \quad-\min \left(\widehat{S}_{i(\tau-1) n}-\sum_{\substack{n^{\prime}=1, n^{\prime} \neq n}}^{N} X_{i(\tau-1) n n^{\prime}}, \mathrm{LD} \cdot d_{i(\tau-1) n}\right) .
\end{aligned}
$$

After calculating $S_{i \tau n}$, the total sales $\left(\mathrm{TS}^{\prime}\right)$, the total ordering cost (TP), and the total stockout cost $\left(\mathrm{TO}^{\prime}\right)$ could be calculated by (2), (3), and (7), respectively. The total transportation cost (TT) has nothing with lateral transshipment, so it could be calculated still by (4). Here the most difficult thing is to compute the total inventory cost.

The total holding cost $\left(\mathrm{TI}_{s}^{\prime}\right)$ at Supply Hub will not be affected by lateral transshipment and could still be calculated by (5). The total inventory cost $\left(\mathrm{TI}_{d}\right)$ at distributor will change, because the inventory level of the transferor will drop down $X_{i \tau n n^{\prime}}$ and the transferee's inventory level will immediately increase by the amount of $X_{i \tau n n^{\prime}}$ at the transshipment moment which is consumed gradually by the customer's requirement within the lead time. Assume that distributor $n$ is the transferor and distributor $n^{\prime}$ is transferee; the inventory changes at the two sides for the transshipment are shown in Figure 4.

From Figure 4, the variation amount of inventory cost $\Delta \mathrm{TI}$ at distributor is

$$
\Delta \mathrm{TI}=\sum_{i=1}^{I} \sum_{\tau=1}^{M J} \sum_{n=1}^{N} \sum_{\substack{n^{\prime}=1, n^{\prime} \neq n}}^{N}\left(X_{i \tau n n^{\prime}} \cdot \mathrm{LD}-\frac{\left(\left[\widehat{S}_{i \tau n^{\prime}}\right]^{+}+X_{i \tau n n^{\prime}}\right)^{2}-\left(\left[\widehat{S}_{i \tau n^{\prime}}\right]^{+}\right)^{2}}{2 d_{i \tau n}}\right) \cdot h_{i} .
$$




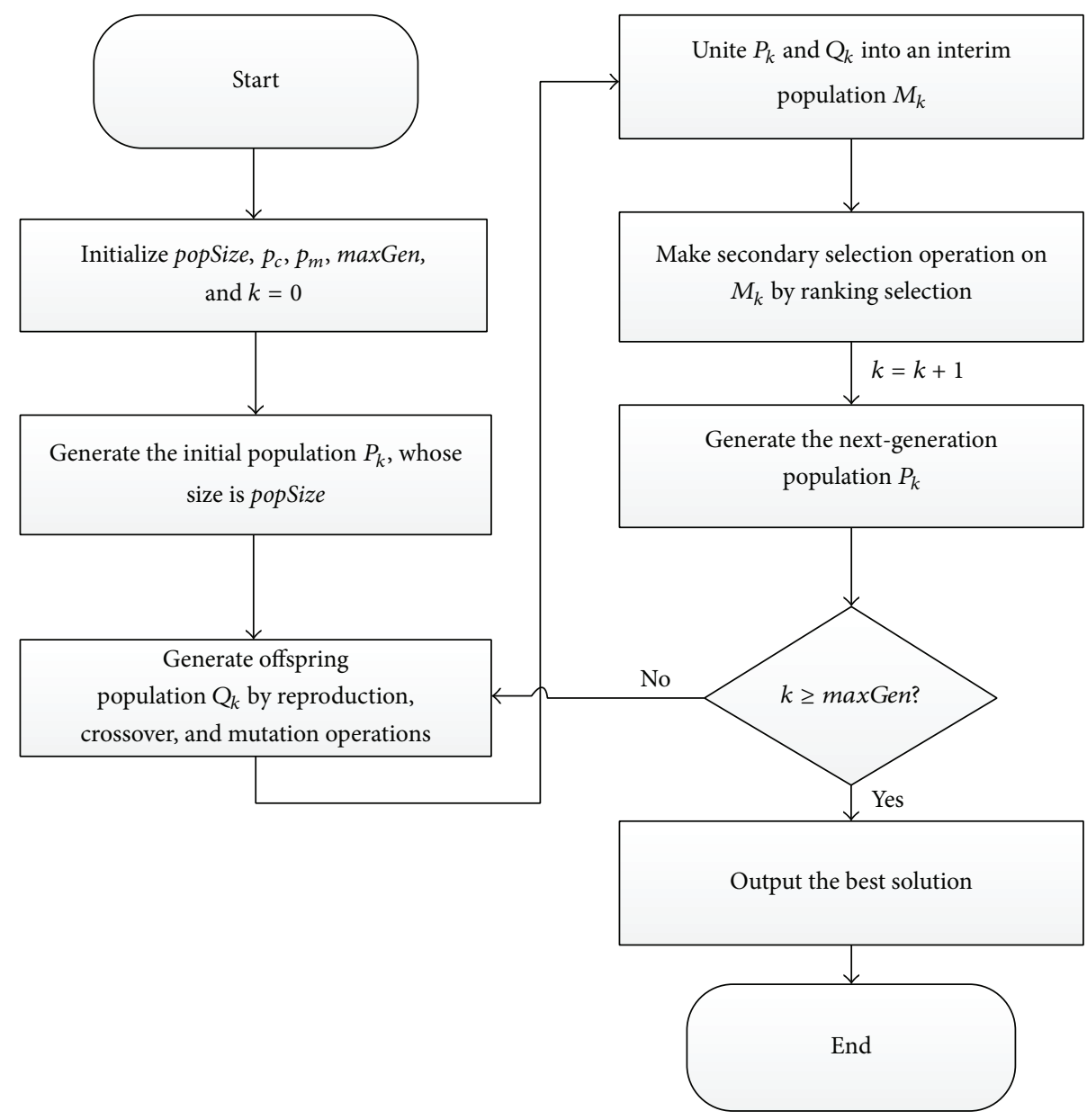

FIGURE 5: Flow chart of the devised genetic algorithm.

The total inventory cost $\mathrm{TI}_{d}^{\prime}$ at distributor is

$$
\mathrm{TI}_{d}^{\prime}=\mathrm{TI}_{d}-\Delta \mathrm{TI}
$$

Consequently, the total profit of supply chain with lateral transshipment is

$$
\mathrm{TV}^{\prime}=\mathrm{TS}^{\prime}-\left(\mathrm{TP}^{\prime}+\mathrm{TT}^{\prime}+\mathrm{TI}_{s}^{\prime}+\mathrm{TI}_{d}^{\prime}+\mathrm{TL}+\mathrm{TO}^{\prime}\right)
$$

\section{The Algorithm to Solve the Model with Lateral Transshipment}

The supply chain system shown in Figure 1 considers Supply Hub and lateral transshipment simultaneously. It is intractable task to solve this model due to the complexity computing transshipment level. Genetic algorithm (GA) is a random search technique and global optimization algorithm based on natural selection and genetic mechanism [42]. Because GA has such advantages like independence of the application domain, suitability for very general optimization problems (no requirements of linearity, convexity, and differentiability), robustness with respect to starting points, and abilities of leaving local optima and finding the global one, it is a good method to solve production and operations management problems [43]. So we select a GA with real number coding scheme to solve the problem, in which two stage selection technology is applied to improve the performance of genetic algorithm. Let popSize represent the population size, maxGen denotes the maximum iteration number, $k$ denotes the number of current generations, $p_{c}$ is the crossover probability, and $p_{m}$ is the mutation probability; then flow chart of the algorithm can be expressed by Figure 5.

We use the real number coding scheme in GA. A chromosome consists of the inventory levels $S_{\text {in }}$ at distributors; for instance,

$$
\begin{aligned}
G= & \left\{S_{11}, S_{12}, \ldots, S_{1 N}, \ldots, S_{i 1}, S_{i 2}, \ldots, S_{i N}, \ldots, S_{I 1}, S_{I 2},\right. \\
& \left.\ldots, S_{I N}\right\} .
\end{aligned}
$$

Obviously, the length of a chromosome is $I \times N$.

We take formula (18) as fitness function; that is, individual fitness represents the total profit $\left(\mathrm{TV}^{\prime}\right)$ of supply chain system.

For the sake of simplicity, let $g$ denote a gene of the chromosome, $G_{k}=\left\{g_{k}^{1}, g_{k}^{2}, \ldots, g_{k}^{I \times N}\right\}$ and $G_{m}=\left\{g_{m}^{1}, g_{m}^{2}, \ldots\right.$, $\left.g_{m}^{I \times N}\right\}$ are the chromosomes selected from parent population, 


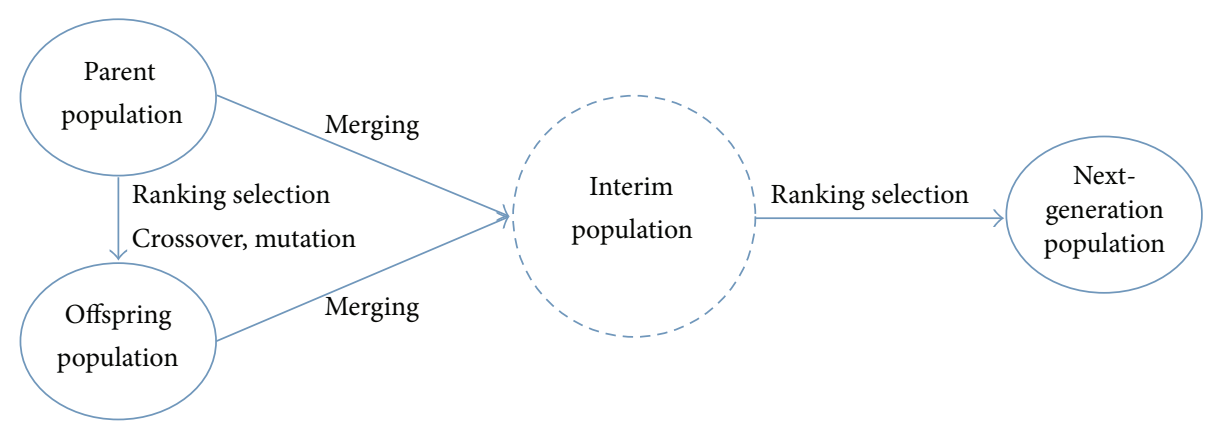

FIGURE 6: Two-stage selection policy.

$l(l=1,2, \ldots, I \times N)$ is the crossover point chosen randomly, $\lambda$ is crossover coefficient which is a random number at the interval $(0,1)$, and $p_{c}$ is crossover probability. Then the single point nonuniform arithmetic crossover operator can be formulated as follows:

$$
\begin{aligned}
& c_{j_{1}}^{l}=\lambda g_{j}^{l}+(1-\lambda) g_{m}^{l} \\
& c_{j_{2}}^{l}=(1-\lambda) g_{j}^{l}+\lambda g_{m}^{l},
\end{aligned}
$$

where $C_{j_{1}}=\left\{c_{j_{1}}^{1}, \ldots, c_{j_{1}}^{i}, \ldots, c_{j_{1}}^{I \times N}\right\}$ and $C_{j_{2}}=\left\{c_{j_{2}}^{1}, \ldots, c_{j_{2}}^{i}, \ldots\right.$, $\left.c_{j_{2}}^{I \times N}\right\}$ are offspring chromosomes.

We adopt uniform mutation policy; namely, a gene is replaced with the value chosen randomly within the value range of the gene.

To prevent the phenomenon in the GA iteration that subsequent crossover and mutation operations may incur the missing of excellent solutions or solution components from parent population, we introduce two-stage selection policy. At the first stage, the algorithm selects individuals from parent population by ranking selection which is used to generate offspring population by genetic operations, and then the parent and offspring populations form a union named interim population. At the second stage, the algorithm carries out a ranking-based selection procedure for the interim population once again to form the next-generation population. For more clarity, we demonstrate the two-stage procedure by Figure 6.

The multi-item distribution model without lateral transshipment is viewed as a comparison benchmark. We apply Lingo software to solve it.

\section{Computation Examples}

6.1. The Description of Example. We consider a three-echelon supply chain composed of two suppliers, Supply Hub and two distributors; namely, $I=2, N=2$. Replenishment cycle at Supply Hub $T=6$, replenishment cycle at distributor $\mathrm{TD}=2$; that is, $M=3$. Parameters of Supply Hub and distributors are shown in Tables 1 and 2, respectively. The demand rates of the product 1 and product 2 comply with uniform distribution of $U(20,30)$ and $U(16,30)$, respectively. The demand rates used by the experiment are shown in Table 3.
TABle 1: The parameters at Supply Hub.

\begin{tabular}{lcc}
\hline Parameters & $i=1$ & Product \\
& \multicolumn{2}{c}{$i=2$} \\
$P_{i}$ & 6.2 & 10.4 \\
$F_{i}$ & 100 & 110 \\
$A_{i}$ & 0.1 & 0.1 \\
$H_{i}$ & 0.1 & 0.1 \\
$\pi_{i}$ & 0.4 & 0.4 \\
\hline
\end{tabular}

TABLE 2: The parameters at distributors.

\begin{tabular}{lcccc}
\hline \multirow{2}{*}{ Parameters } & \multicolumn{4}{c}{ Suppliers } \\
& $i=1$ & $i=2$ & $i=1$ & $i=2$ \\
\hline$V_{\text {in }}$ & 13.4 & 18.6 & 13.6 & 18.8 \\
$f_{\text {in }}$ & 40 & 45 & 50 & 55 \\
$a_{\text {in }}$ & 0.1 & 0.15 & 0.1 & 0.15 \\
$h_{i}$ & 0.4 & 0.6 & 0.4 & 0.6 \\
\hline
\end{tabular}

TABLE 3: The demand rates at distributors.

\begin{tabular}{lccc}
\hline & $\tau=1$ & $\tau=2$ & $\tau=3$ \\
\hline$d_{11}$ & 26 & 20 & 29 \\
$d_{12}$ & 24 & 26 & 25 \\
$d_{21}$ & 17 & 27 & 21 \\
$d_{22}$ & 29 & 18 & 28 \\
\hline
\end{tabular}

6.2. The Effect of Two-Stage Selection Technology. In this experiment, the crossover and mutation probabilities, respectively, take the value of 0.75 and 0.05 ; the population size is set to be 50 . Through many experiments, we find that the maximum convergence generation of the proposed GA is 50 . Figure 7 shows the convergence of the algorithm in a running.

To test the effect of two-stage selection, the GA-based algorithms with and without two-stage selection are carried out 20 times where the terminate conditions are set to be 50 generations. The results are shown in Table 4.

From Table 4, two-stage selection technology has little effect on the run time; it takes about $50 \mathrm{~ms}$ for the two algorithms to run 50 iterations. As a whole, the algorithm with two-stage selection would converge at around 20 generations. 
TABLE 4: The effect of two-stage selection technology.

\begin{tabular}{|c|c|c|c|c|}
\hline \multirow{2}{*}{ Number of experiments } & \multicolumn{2}{|c|}{ Without two-stage selection } & \multicolumn{2}{|c|}{ With two-stage selection } \\
\hline & Run time (ms) & Convergence & Run time (ms) & Convergence \\
\hline 1 & 55 & $*$ & 52 & 14 \\
\hline 2 & 51 & * & 50 & 23 \\
\hline 3 & 53 & * & 45 & 9 \\
\hline 4 & 50 & 9 & 51 & 19 \\
\hline 5 & 51 & $*$ & 50 & 15 \\
\hline 6 & 54 & $*$ & 51 & 12 \\
\hline 7 & 56 & 27 & 50 & 14 \\
\hline 8 & 50 & $*$ & 50 & 16 \\
\hline 9 & 49 & $*$ & 50 & 22 \\
\hline 10 & 52 & 34 & 52 & 14 \\
\hline 11 & 51 & $*$ & 51 & 13 \\
\hline 12 & 49 & 46 & 51 & 17 \\
\hline 13 & 51 & $*$ & 50 & 25 \\
\hline 14 & 50 & 7 & 51 & 20 \\
\hline 15 & 54 & 19 & 53 & 13 \\
\hline 16 & 52 & 26 & 56 & 7 \\
\hline 17 & 51 & $*$ & 51 & 17 \\
\hline 18 & 51 & $*$ & 52 & 7 \\
\hline 19 & 53 & $*$ & 50 & 21 \\
\hline 20 & 51 & 32 & 54 & 15 \\
\hline
\end{tabular}

${ }^{*}$ Representing that the run is not convergent within 50 generations.

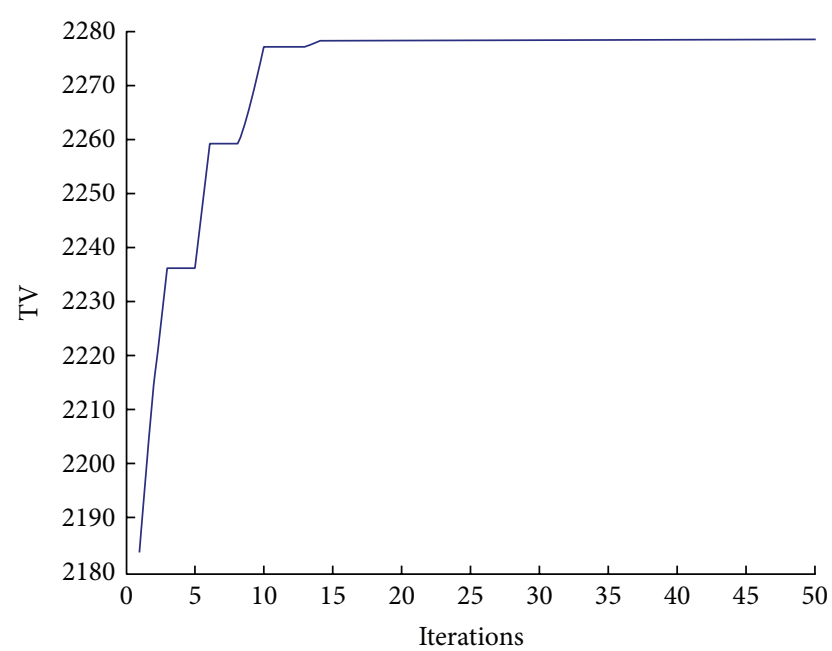

FIgURE 7: The convergence of the GA-based algorithm.

On the contrary, in the 20 experiments for the algorithm without two-stage selection, only 8 experiments are convergent within 50 generations. Obviously, the two-stage selection technology can boost or accelerate the convergence of the algorithm.

6.3. The Effect of Lateral Transshipment. For the model considering lateral transshipment, the optimal solution obtained by GA is $S_{11}=52, S_{12}=48, S_{21}=54$, and $S_{21}=59$; the total profit of the supply chain $\mathrm{TV}=2277.2$. Under
TABLE 5: The detailed experimental results without and with lateral transshipment.

\begin{tabular}{lcc}
\hline & $\begin{array}{c}\text { Without lateral } \\
\text { transshipment }\end{array}$ & $\begin{array}{c}\text { With lateral } \\
\text { transshipment }\end{array}$ \\
\hline$S_{11}$ & 52 & 52 \\
$S_{12}$ & 42 & 48 \\
$S_{21}$ & 50 & 54 \\
$S_{22}$ & 57 & 59 \\
TP & 5866.8 & 6165.6 \\
TT & 597.5 & 605.0 \\
TI & 378.6 & 403.9 \\
TS & 9010.8 & 9520.8 \\
TO & 117.4 & 67.4 \\
TV & 2050.5 & 2277.2 \\
\hline
\end{tabular}

the same parameters settings, we use Lingo to solve the model without transshipment, the results are $S_{11}=52, S_{12}=42$, $S_{21}=50$, and $S_{21}=57$, and the total profit of the supply chain TV $=2050.5$. Comparing with the two results, we find that the total profit of the supply chain grows by $11.1 \%$ in the case of lateral transshipment. Table 5 shows the concrete comparisons of the experimental results.

According to Table 5, the maximal stock levels of the case with lateral transshipment are higher than that of the case without lateral transshipment. This leads to the increase of the total order quantity and the corresponding growth of the ordering, transportation, and inventory costs. However, 
TABLE 6: The computation results related to product 1.

\begin{tabular}{|c|c|c|c|c|c|c|c|c|c|c|}
\hline \multirow{3}{*}{ Period $\tau$} & \multicolumn{10}{|c|}{ Distributor $n$} \\
\hline & \multicolumn{2}{|c|}{$\begin{array}{l}\text { Demand within } \\
\text { period } \tau(\mathrm{TD})\end{array}$} & \multicolumn{2}{|c|}{$\begin{array}{l}\text { Initial inventory } \\
\text { level at period } \tau\end{array}$} & \multicolumn{2}{|c|}{$\begin{array}{l}\text { Shortage level } \\
\text { within the period } \tau\end{array}$} & \multicolumn{2}{|c|}{$\begin{array}{l}\text { Shortage level } \\
\text { within lead time }\end{array}$} & \multicolumn{2}{|c|}{ Transshipment level } \\
\hline & $n=1$ & $n=2$ & $n=1$ & $n=2$ & $n=1$ & $n=2$ & $n=1$ & $n=2$ & $1 \rightarrow 2$ & $2 \rightarrow 1$ \\
\hline \multicolumn{11}{|c|}{ Without transshipment } \\
\hline 1 & 52 & 34 & 52 & 42 & 0 & 0 & 0 & 0 & 0 & 0 \\
\hline 2 & 40 & 54 & 50 & 41 & 0 & 13 & 0 & 3 & 0 & 0 \\
\hline 3 & 58 & 42 & 50 & 42 & 8 & 0 & 2 & 0 & 0 & 0 \\
\hline \multicolumn{11}{|c|}{ With transshipment } \\
\hline 1 & 52 & 34 & 52 & 48 & 0 & 0 & 0 & 0 & 0 & 0 \\
\hline 2 & 40 & 54 & 50 & 47 & 0 & 4 & 0 & 0 & 3 & 0 \\
\hline 3 & 58 & 42 & 50 & 46 & 6 & 0 & 0 & 0 & 0 & 2 \\
\hline
\end{tabular}

lateral transshipment could decrease the total shortage level and the total stockout cost of the supply chain system. At the same time, it makes the total sale volume increase. Since the profit growth exceeds the cost increase, we see that the total profit of the former is higher than that of the latter.

In order to make a comprehensive display and thorough analysis of the calculation results, we separate out the calculation results related to product 1 , which is shown in Table 6. Due to the uncertainty of demand, the phenomenon often appears in that the inventory of a distributor is surplus while one of another distributor is insufficient. For example, in period 2, distributor 1 possesses beginning inventory of 50 units, but it merely faces the demand of 40 units, which means there are 10 units left. However, at the same period, distributor 2 is of beginning inventory of 41 units, and it needs to meet the demand of 54 units, which means there is shortage level of 13 units. Hence, if lateral transshipment between distributors is allowed, the stock at distributors could be rebalanced, and then the shortage possibility and total inventory level for the system both will be reduced. The conclusion has been verified by the data in Table 6; namely, the shortage level within the replenishment lead time drops down to zero owing to lateral transshipment. But for lateral transshipment that is allowed only at the moment (TD - LD), this results in the fact that the shortage level before the moment (TD - LD) is coped with only by increasing the order quantity and inventory level. For instance, the initial inventory level of product 1 at distributor 2 under the transshipping case is clearly higher than the one for the no transshipment case. If the transshipment rule is changed to judging whether lateral transshipment is adopted once a distributor is out of stock, the shortage level and cost can be decreased further.

In order to further verify the influence of lateral transshipment on the system total profit, we randomly generate 49 sets of requirements according to the demand distribution stated at the fore. The experimental results are shown in Figure 8.

From Figure 8, we can see that in 49 groups of comparison experiments, the total profit of supply chain with lateral transshipment is apparently higher than that of supply chain with no transshipment. The profit difference varies up to $16.2 \%$ and

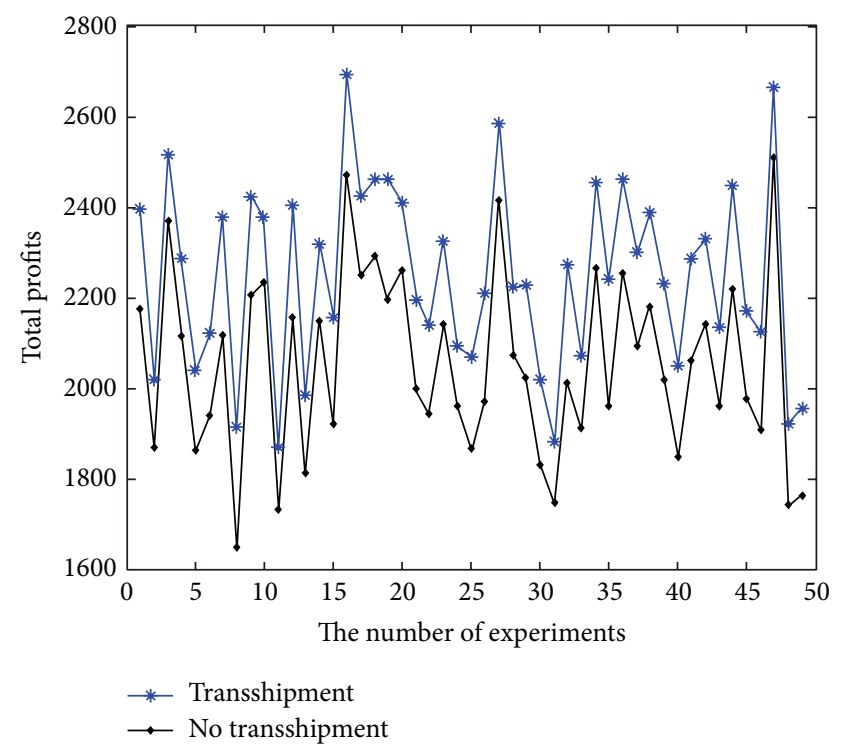

Figure 8: The effect of lateral transshipment on the system total profit.

down to $6.1 \%$. Thus, the policy of lateral transshipment has obvious optimization effect on the total profit of supply chain with Supply Hub under uncertain demand.

\section{Conclusion}

This paper concentrated on the supply chain comprising multisupplier, Supply Hub, and multidistributor, established multi-item distribution model with lateral transshipment. To the best of our knowledge, this is the first study on the multilocation inventory system with Supply Hub and lateral transshipment. For purposes of comparison, we built the maximal total profit model of the inventory system, respectively, for the cases without transshipment and with transshipment. The former is viewed as a comparison object and is easy to solve, so we adopted Lingo software to obtain its exact solution. The latter is difficult to solve in view of 
the transshipment, and we devised a GA-based solution algorithm with two-stage selection technology. In order to verify the effectiveness and efficiency of the proposed model and solve algorithm, we presented the comparison experiment. By means of the experiment, we firstly showed that the two-stage selection technology could boost or accelerate the convergence of the algorithm. Secondly, we verified that the policy of lateral transshipment could improve the performance of the multilocation inventory system with Supply Hub. From the experiment, we also found that the system performance could be further improved if transshipment policy was immediately considered once a distributor is out of stock. This will be one of the future works of this research. In addition, another valuable future direction of this paper is to consider multiple product distribution problems with Supply Hub and lateral transshipment under other demand distributions.

\section{Conflict of Interests}

The authors declare that there is no conflict of interests regarding the publication of this paper.

\section{Acknowledgments}

This research was supported by National Natural Science Foundation of China (71171072). The authors are thankful to two anonymous referees and the editor for their constructive comments, which resulted in the subsequent improvement of this paper.

\section{References}

[1] L. E. Cárdenas-Barrón, "Optimizing inventory decisions in a multi-stage multi-customer supply chain: a note," Transportation Research Part E: Logistics and Transportation Review, vol. 43, no. 5, pp. 647-654, 2007.

[2] L. E. Cárdenas-Barrón, G. Treviño-Garza, and H. M. Wee, "A simple and better algorithm to solve the vendor managed inventory control system of multi-product multi-constraint economic order quantity model," Expert Systems with Applications, vol. 39, no. 3, pp. 3888-3895, 2012.

[3] A. Zuckerman, "Compaq switches to pre-position inventory model," World Trade, vol. 13, no. 4, pp. 72-74, 2000.

[4] E. Barnes, J. Dai, S. Deng, D. Down, M. Goh, and L. H. Chuin, "On the strategy of supply hubs for cost reduction and responsiveness," The ICFAI Journal of Operations Management, pp. 1-27, 2003.

[5] L. R. Kopczak, Apple Computer's Supplier Hubs: A Tale of Three Cities, Stanford Global Supply Chain Management Forum Case, Stanford, Calif, USA, 1997.

[6] J. Shah and M. Goh, "Setting operating policies for supply hubs," International Journal of Production Economics, vol. 100, no. 2, pp. 239-252, 2006.

[7] R. Gaonkar and N. Visvanadham, "Collaborative scheduling model for supply-hub management," in Proceedings of the $3 \mathrm{rd}$ AEGEAN International conference on Analysis and Modeling of Manufacturing Systems, pp. 1-6, 2001.

[8] J. Li, S. Ma, P. Guo, and Z. Zuo, "Analysis on design of supply chain embedding supply-hub with BOM," in Proceedings of the 4th International Conference on Wireless Communications, Networking and Mobile Computing (WiCOM '08), pp. 1-4, IEEE, Dalian, China, October 2008.

[9] J. Li, N. Xiong, L. Sun, A. Yuan, J. Chen, and M. Cao, "Supply chain design model based on multi-supply hubs," in Proceedings of the 12th International Conference on Computational Science and Engineering (CSE '09), vol. 1, pp. 449-454, August 2009.

[10] K. Huang and S.-H. Ma, "Coordinated replenishment with information sharing between two suppliers based on supplyhub," in Proceedings of the 17th International Conference on Management Science and Engineering (ICMSE '10), pp. 403-409, November 2010

[11] Q. G. Liu and J. X. Chen, "A coordination mechanism of supply chain under asymmetric information based on supply hub," in Proceedings of the International Conference on E-Product E-Service and E-Entertainment (ICEEE '10), pp. 1-4, Henan, China, November 2010.

[12] W. Lin, "An effective lean supply inventory management model using VMI hub," in Proceedings of the 18th IEEE International Conference on Industrial Engineering and Engineering Management (IEEM '11), pp. 950-954, December 2011.

[13] X. Qiu and G. Q. Huang, "Supply Hub in Industrial Park (SHIP): the value of freight consolidation," Computers \& Industrial Engineering, vol. 65, no. 1, pp. 16-27, 2013.

[14] X. Qiu, H. Luo, G. Xu, R. Zhong, and G. Q. Huang, "Physical assets and service sharing for IoT-enabled Supply Hub in Industrial Park (SHIP)," International Journal of Production Economics, vol. 159, pp. 4-15, 2015.

[15] K. S. Krishnan and V. R. K. Rao, "Inventory control in N warehouses," Journal of Industrial Engineering, vol. 16, no. 3, pp. 212-215, 1965.

[16] C. C. Chiou, "Transshipment problems in supply chain systems: review and extensions," in Supply Chain, Theory and Applications, V. Kordic, Ed., pp. 558-579, I-Tech Education and Publishing, Vienna, Austria, 2008.

[17] C. Paterson, G. Kiesmüller, R. Teunter, and K. Glazebrook, "Inventory models with lateral transshipments: a review," European Journal of Operational Research, vol. 210, no. 2, pp. 125-136, 2011.

[18] X. Huang, "A review on policies and supply chain relationships under inventory transshipment," Bulletin of Statistics and Operations Research, vol. 29, no. 1, pp. 21-42, 2013.

[19] T. W. Archibald, S. A. E. Sassen, and L. C. Thomas, "An optimal policy for a two depot inventory problem with stock transfer," Management Science, vol. 43, no. 2, pp. 173-183, 1997.

[20] A. A. Kranenburg and G. J. van Houtum, "A new partial pooling structure for spare parts networks," European Journal of Operational Research, vol. 199, no. 3, pp. 908-921, 2009.

[21] H. Wong, G. J. van Houtum, D. Cattrysse, and D. van Oudheusden, "Simple, efficient heuristics for multi-item multi-location spare parts systems with lateral transshipments and waiting time constraints," Journal of the Operational Research Society, vol. 56, no. 12, pp. 1419-1430, 2005.

[22] H. Wong, G. J. van Houtum, D. Cattrysse, and D. van Oudheusden, "Multi-item spare parts systems with lateral transshipments and waiting time constraints," European Journal of Operational Research, vol. 171, no. 3, pp. 1071-1093, 2006.

[23] R. A. Shumsky and F. Zhang, "Dynamic capacity management with substitution," Operations Research, vol. 57, no. 3, pp. 671684, 2009. 
[24] Z. M. Avsar and M. Baykal-Gürsoy, "Inventory control under substitutable demand: a stochastic game application," Naval Research Logistics, vol. 49, no. 4, pp. 359-375, 2002.

[25] S. Netessine and N. Rudi, "Centralized and competitive inventory models with demand substitution," Operations Research, vol. 51, no. 2, pp. 329-335, 2003.

[26] B. Rottkemper, K. Fischer, and A. Blecken, "A transshipment model for distribution and inventory relocation under uncertainty in humanitarian operations," Socio-Economic Planning Sciences, vol. 46, no. 1, pp. 98-109, 2012.

[27] E. M. Alvarez, M. C. van der Heijden, I. M. H. Vliegen, and W. H. M. Zijm, "Service differentiation through selective lateral transshipments," European Journal of Operational Research, vol. 237, no. 3, pp. 824-835, 2014.

[28] M. Moghaddam and S. Y. Nof, "Combined demand and capacity sharing with best matching decisions in enterprise collaboration," International Journal of Production Economics, vol. 148, pp. 93-109, 2014.

[29] L. A. Michaelraj and P. Shahabudeen, "Replenishment policies for sustainable business development in a continuous credit based vendor managed inventory distribution system," Computers \& Industrial Engineering, vol. 56, no. 1, pp. 260-266, 2009.

[30] H. Lan, R. Li, Z. Liu, and R. Wang, "Study on the inventory control of deteriorating items under VMI model based on bilevel programming," Expert Systems with Applications, vol. 38, no. 8, pp. 9287-9295, 2011.

[31] S. P. Nachiappan and N. Jawahar, "A genetic algorithm for optimal operating parameters of VMI system in a two-echelon supply chain," European Journal of Operational Research, vol. 182, no. 3, pp. 1433-1452, 2007.

[32] G. Sue-Ann, S. G. Ponnambalam, and N. Jawahar, "Evolutionary algorithms for optimal operating parameters of vendor managed inventory systems in a two-echelon supply chain," Advances in Engineering Software, vol. 52, pp. 47-54, 2012.

[33] A. Diabat, "Hybrid algorithm for a vendor managed inventory system in a two-echelon supply chain," European Journal of Operational Research, vol. 238, no. 1, pp. 114-121, 2014.

[34] J. Sadeghi, S. Sadeghi, and S. T. A. Niaki, "A hybrid vendor managed inventory and redundancy allocation optimization problem in supply chain management: an NSGA-II with tuned parameters," Computers \& Operations Research, vol. 41, no. 1, pp. 53-64, 2014.

[35] J. Sadeghi and S. T. Niaki, "Two parameter tuned multiobjective evolutionary algorithms for a bi-objective vendor managed inventory model with trapezoidal fuzzy demand," Applied Soft Computing, vol. 30, pp. 567-576, 2015.

[36] F. T. S. Chan, S. H. Chung, and S. Wadhwa, "A hybrid genetic algorithm for production and distribution," Omega, vol. 33, no. 4, pp. 345-355, 2005.

[37] A. K. Maiti, A. K. Bhunia, and M. Maiti, "An application of realcoded genetic algorithm (RCGA) for mixed integer non-linear programming in two-storage multi-item inventory model with discount policy," Applied Mathematics and Computation, vol. 183, no. 2, pp. 903-915, 2006.

[38] S. H. R. Pasandideh, S. T. A. Niaki, and N. Tokhmehchi, "A parameter-tuned genetic algorithm to optimize two-echelon continuous review inventory systems," Expert Systems with Applications, vol. 38, no. 9, pp. 11708-11714, 2011.

[39] W. Yang, F. T. S. Chan, and V. Kumar, "Optimizing replenishment polices using Genetic Algorithm for single-warehouse multi-retailer system," Expert Systems with Applications, vol. 39, no. 3, pp. 3081-3086, 2012.
[40] B. Paul and C. Rajendran, "Rationing mechanisms and inventory control-policy parameters for a divergent supply chain operating with lost sales and costs of review," Computers \& Operations Research, vol. 38, no. 8, pp. 1117-1130, 2011.

[41] X. Chen, G. Hao, X. Li, and K. F. C. Yiu, "The impact of demand variability and transshipment on vendor's distribution policies under vendor managed inventory strategy," International Journal of Production Economics, vol. 139, no. 1, pp. 42-48, 2012.

[42] J. H. Holland, Adaptation in Natural and Artificial Systems, The University of Michigan Press, Ann Arbor, Mich, USA, 1975.

[43] H. Aytug, M. Khouja, and F. E. Vergara, "Use of genetic algorithms to solve production and operations management problems: a review," International Journal of Production Research, vol. 41, no. 17, pp. 3955-4009, 2003. 


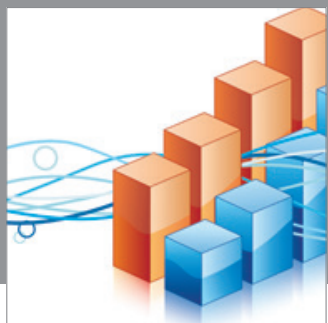

Advances in

Operations Research

mansans

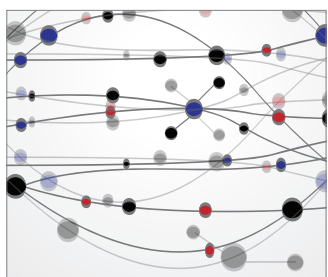

The Scientific World Journal
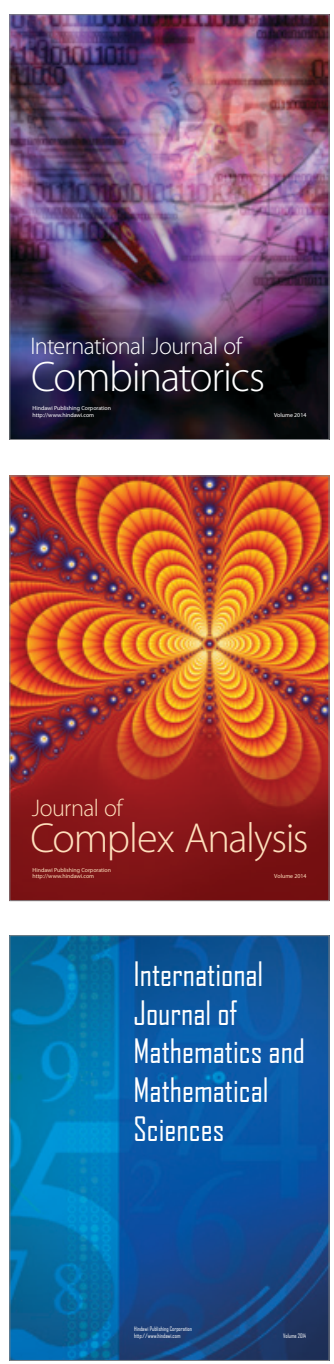
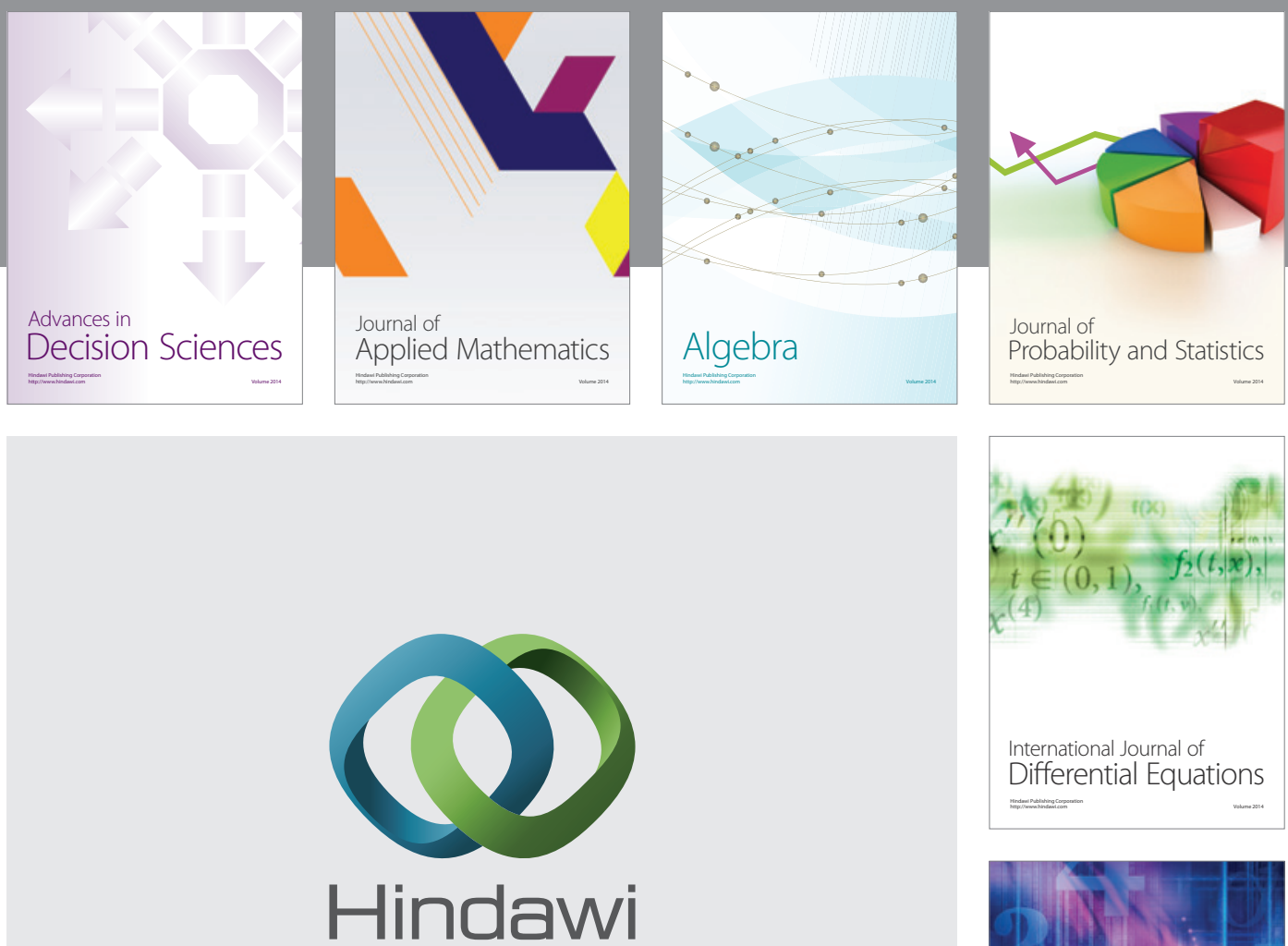

Submit your manuscripts at http://www.hindawi.com
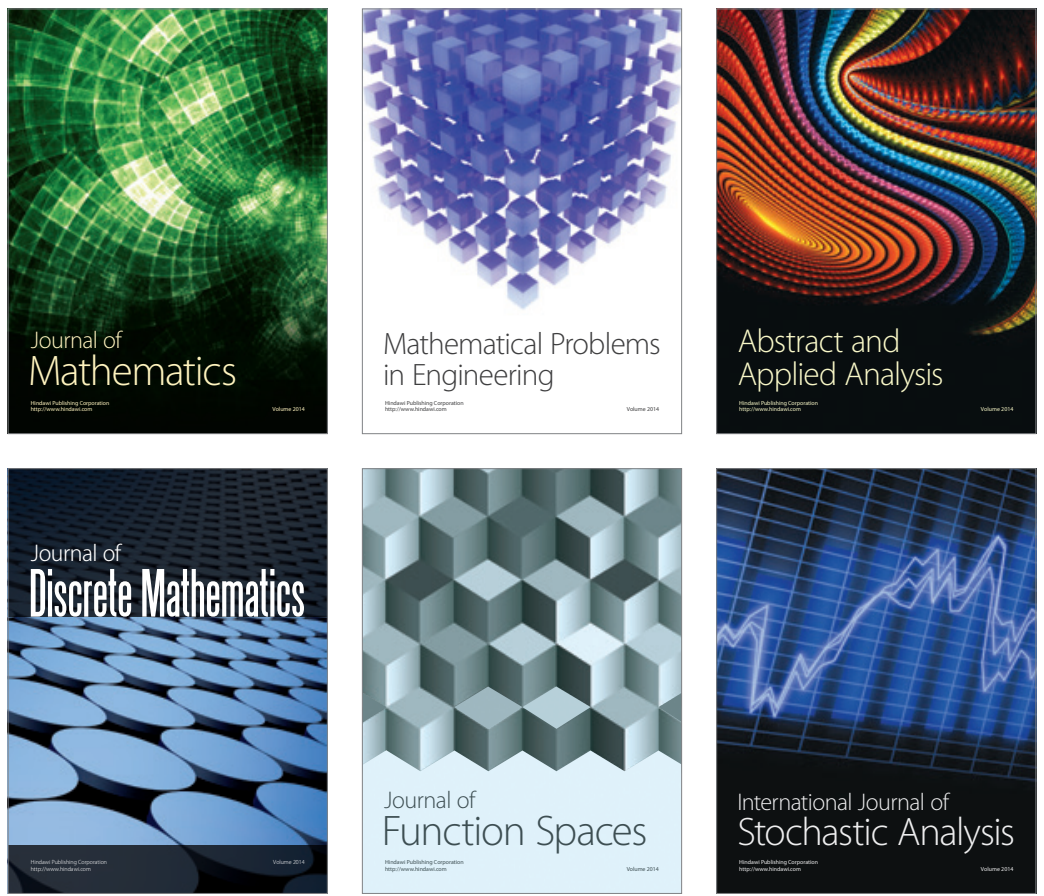

Journal of

Function Spaces

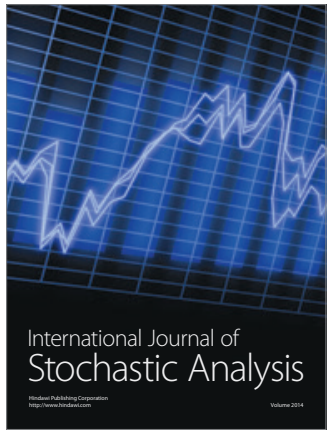

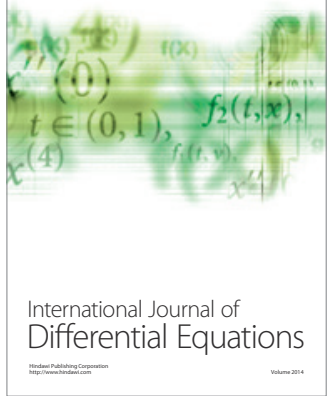
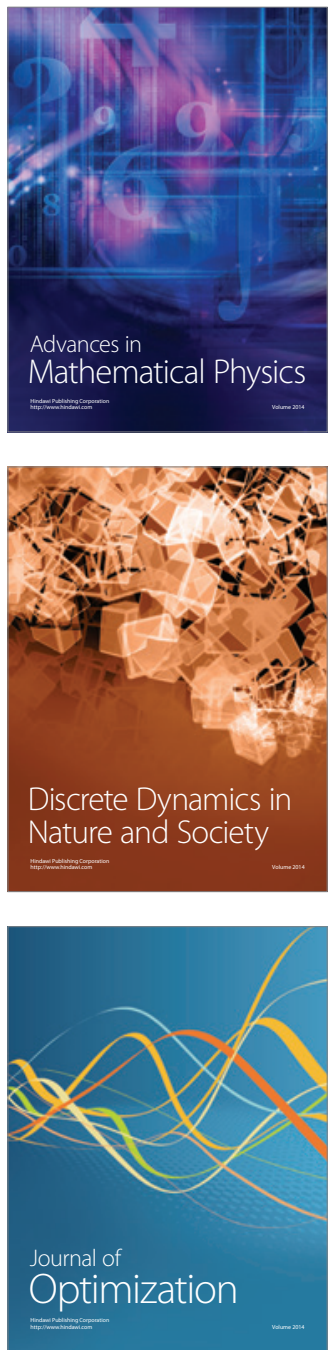